OPEN ACCESS

Edited by:

Pei-Ji Liang,

Shanghai Jiao Tong University, China

Reviewed by:

Erwei Yin,

China Astronaut Research and

Training Center, China

Radwa Khalil,

Jacobs University Bremen, Germany

*Correspondence:

Qi Li

liqi@cust.edu.cn

Received: 14 May 2019

Accepted: 20 December 2019

Published: 15 January 2020

Citation:

Lu Z, Li Q, Gao N and Yang J (2020)

The Self-Face Paradigm Improves the

Performance of the

P300-Speller System.

Front. Comput. Neurosci. 13:93.

doi: 10.3389/fncom.2019.00093

\section{The Self-Face Paradigm Improves the Performance of the P300-Speller System}

\author{
Zhaohua Lu, Qi Li*, Ning Gao and Jingjing Yang \\ School of Computer Science and Technology, Changchun University of Science and Technology, Changchun, China
}

Objective: Previous studies have shown that the performance of the famous face P300-speller was better than that of the classical row/column flashing P300-speller. Furthermore, in some studies, the brain was more active when responding to one's own face than to a famous face, and a self-face stimulus elicited larger amplitude event-related potentials (ERPs) than did a famous face. Thus, we aimed to study the role of the self-face paradigm on further improving the performance of the P300-speller system with the famous face P300-speller paradigm as the control paradigm.

Methods: We designed two facial P300-speller paradigms based on the self-face and a famous face (Ming Yao, a sports star; the famous face spelling paradigm) with a neutral expression.

Results: ERP amplitudes were significantly greater in the self-face than in the famous face spelling paradigm at the parietal area from 340 to $480 \mathrm{~ms}$ (P300), from 480 to $600 \mathrm{~ms}$ (P600f), and at the fronto-central area from 700 to $800 \mathrm{~ms}$. Offline and online classification results showed that the self-face spelling paradigm accuracies were significantly higher than those of the famous face spelling paradigm at superposing first two times $(P<$ 0.05). Similar results were found for information transfer rates $(P<0.05)$.

Conclusions: The self-face spelling paradigm significantly improved the performance of the P300-speller system. This has significant practical applications for brain-computer interfaces (BCls) and could avoid infringement issues caused by using images of other people's faces.

Keywords: brain-computer interface (BCI), event-related potential, famous face, P300-speller, self-face

\section{INTRODUCTION}

A brain-computer interface (BCI) is a communication technology based on brain activity. BCIs allow severely disabled patients, especially patients with amyotrophic lateral sclerosis, to send messages or control external devices without physical actions (Thompson et al., 2013; Rosenfeld and Wong, 2017; Lazarou et al., 2018). BCIs can also help restore function in patients with severe motor disabilities, including patients with spinal cord injury, stroke, neuromuscular disorder, and limb amputation (Takeuchi et al., 2015; Carelli et al., 2017; Wang et al., 2019). In recent years, some studies have used BCIs for enhancing clinical communication assessments in patients with disorders of consciousness (Wang et al., 2017; Jeunet et al., 2018). BCIs are commonly based on electroencephalogram (EEG) that is recorded non-invasively via electrodes placed on the surface of the head (Waldert, 2016). 
The P300 event-related potential (ERP) induced by an oddball paradigm is commonly used in non-invasive BCI systems (Bernat et al., 2001). Farwell and Donchin (1988) first applied the P300 potential to a BCI system; they achieved a character-spelling system based on the P300, which was called the P300-speller system. The users attend to a cell of the matrix (that is, a target character) and count the number of times it is intensified. In this system, the probability of the intensified row/column containing the target character is $1 / 6$ (a matrix of 6 rows and 6 columns), which is an oddball event, which therefore would induce P300 potentials; the system can then output a character by analyzing the P300 potentials. However, the system was not satisfactory due to its low speed and variable accuracy.

A number of studies have attempted to design different paradigms to improve the performance of the P300-speller system (Allison and Pineda, 2003, 2006; Sellers et al., 2006; Salvaris and Sepulveda, 2009; Li et al., 2019). Kaufmann et al. (2011) introduced the famous face paradigm into the P300speller system and found that its performance was markedly superior to that of the conventional P300-speller system, because the face stimulus also induced other ERPs (e.g., the N170) in addition to an increased P300 amplitude, which enhanced the waveform difference between the target and nontarget characters. Subsequently, Jin et al. (2012) compared the performance of P300-speller system between the stimulus types involving a famous face, character flashing, and character movement, and the results showed that the system performed significantly better under the famous face condition than under the other two conditions. Recently, Speier et al. (2017) compared the stimulus types in an online classification of the P300speller, and the results showed that famous faces stimuli yielded superior results than that with both standard and character inversion stimuli. Some researchers have attempted to optimize the face paradigm to improve the performance of the P300speller system. For example, Jin et al. (2014b) designed a new stimulus presentation based on facial expression changes, to reduce adjacent interference annoyance and fatigue. Li et al. (2015) combined chromatic properties and the famous face spelling paradigm, which improved the performance of the P300speller system.

Studies on human face recognition have shown that the brain has specialized cognitive processing for one's own face as compared with other faces. When participants searched for their own face vs. another face, they consistently processed their own face faster than other faces (Tong and Nakayama, 1999). Prior fMRI studies have shown that neural activity was enhanced over the frontal central area for self-face recognition as compared to other face recognition (Kircher et al., 2001). Some ERP studies on human face recognition have shown that the self-face induced greater ERP amplitudes than did other faces. The P300 is more sensitive to the self-face than to other faces (Ninomiya et al., 1998). For example, several studies have found that one's own face elicits a larger P300 amplitude than does a famous face (Caharel et al., 2005; Sui et al., 2006; Miyakoshi et al., 2008; Keyes et al., 2010; Tacikowski et al., 2011). The N170 is face-specific component that reflects facial perception (Bentin and Deouell, 2000; Schweinberger et al., 2002; Herzmann et al., 2004; Carbon et al., 2005). In Caharel et al.'s (2005) study on face processing, the self-face induced a larger N170 amplitude than did famous and unknown faces, distinguishing the self-face from famous and unknown faces. Other studies have also found that the self-face induced a larger N170 amplitude than did other faces (Miyakoshi et al., 2008; Keyes et al., 2010).

Thus, existing studies of face recognition have suggested that the brain is more active in response to the self-face than to a famous face. In the present study, we designed a new spelling paradigm based on self-face stimuli, in which we replaced the famous face with the self-face, to investigate whether the use of the self-face could improve the performance of the P300speller system. The control paradigm was that of the famous face spelling paradigm. We analyzed the ERP waveforms induced in the self-face and famous face spelling paradigms and compared the classification accuracies between the two spelling paradigms.

\section{MATERIALS AND METHODS}

\section{Subjects}

A total of 20 subjects participated in the study; of these, one group ( $n=10$, three men, aged 20-28 years, mean 24.4 years) participated in the offline experiment, and the other group ( $n=10$, six men, aged 22-29 years, mean 25.6 years) participated in the online experiment. The subjects did not have any known neurological disorders and had a normal or corrected-to-normal vision. This study was carried out in accordance with the recommendations of the ethics committee of Changchun University of Science and Technology, which approved the protocol. All subjects gave written informed consent in accordance with the Declaration of Helsinki. All subjects were native Chinese speakers but were familiar with the Western characters used in the display.

\section{Spelling Paradigms}

We designed two P300-speller paradigms based on the conventional P300-speller paradigm. For each paradigm, 36 characters were presented in a $6 \times 6$ matrix subtended at a $13.4^{\circ} \times 19.4^{\circ}(24 \times 1.5 \mathrm{~cm})$ visual angle on a 19 -in screen with a refresh rate of $60 \mathrm{~Hz}$ (Figure 1). In the first paradigm, the rows or columns of the characters were covered with pictures of the subject's self-face while they were intensified (self-face spelling paradigm, as shown in Figure 1; the subject has provided permission to publish his facial photograph in Figure 1). In the control spelling paradigm (the famous face spelling paradigm), the characters were covered with the famous face, and the paradigm's setup was the same as that of the self-face spelling paradigm.

We chose a picture of Ming Yao, a sports star, as the famous face. The subjects' self-face was photographed with a digital camera for the self-face paradigm. All facial images were frontal and showed a neutral expression. These photographs were processed to remove the background and everything below the neck in Adobe Photoshop (Adobe Systems, Inc. San Jose, CA, USA).

In our study, the characters were intensified according to the rows and columns of a virtual matrix (Figure 1, right). In the 

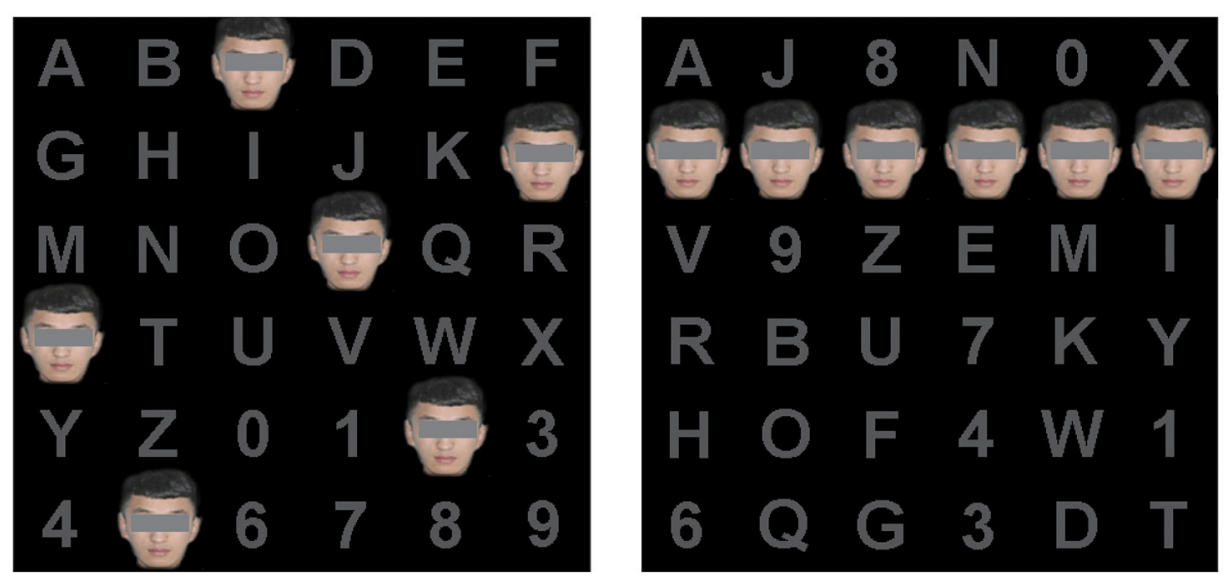

FIGURE 1 | The spelling paradigm. The left figure is the actual spelling matrix, and the right figure is a virtual matrix of the spelling paradigm. The figure shows the self-face paradigm in which the facial photograph is that of a subject.

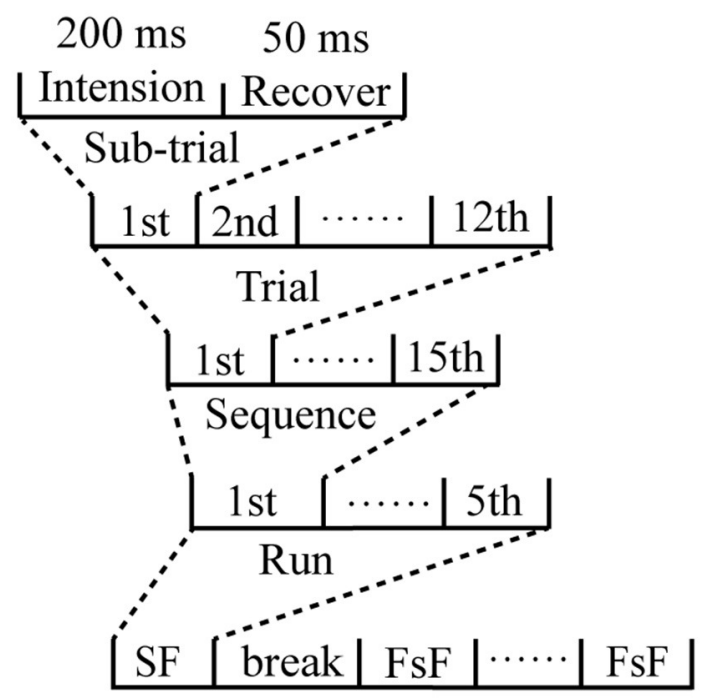

FIGURE 2 | Diagrammatic representation of the time-course of the experiment.

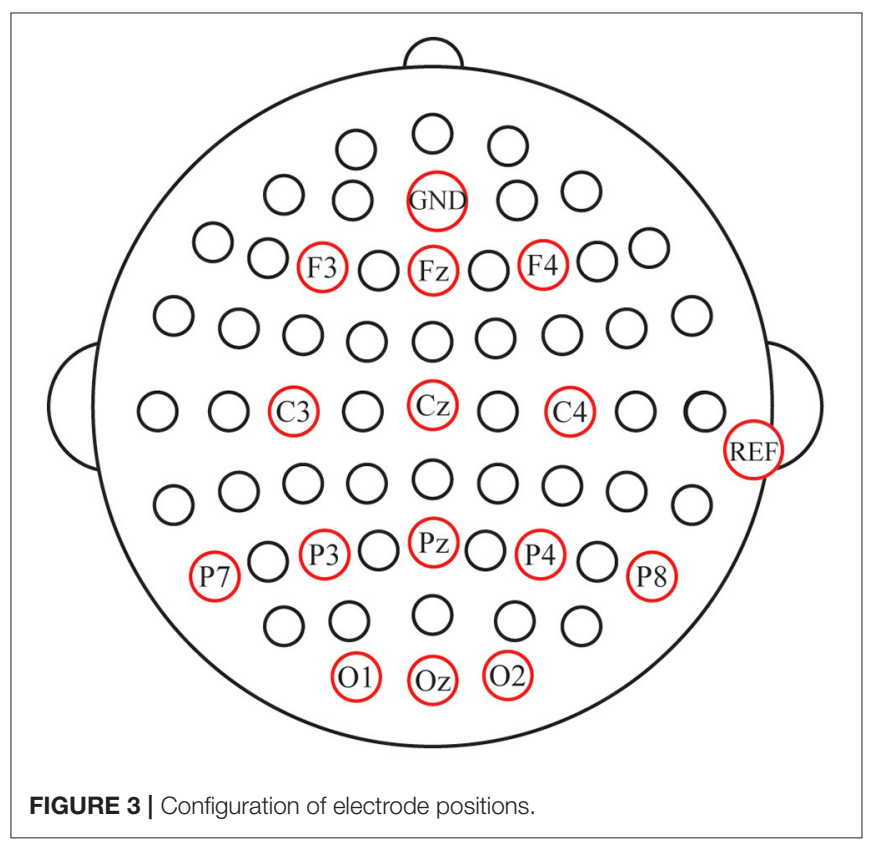

acquisition, subjects were asked to relax and avoid unnecessary movement. The subjects' task was to focus on the target character and silently count the number of times the target characters were covered with faces during stimulus presentation.

In the offline experiment, one flash of a row or column was referred to as a sub-trial. The flash of a row or column that included the target character was defined as a target sub-trial, and the flash of a row or column without the target character was defined as a non-target sub-trial. Six rows and six columns flashed once (12 flashes) as a trial, and the trial was repeated 15 times as a sequence. Thus, each sequence consisted of 180 flashes of rows or columns to output a target character. During the experiment, each spelling paradigm was conducted four times, and each time, a five-character word was spelled out, which was 
considered a run (Figure 2). The runs of the two paradigms were counted alternately to control for potential habituation effects. Participants were allowed to take a 5-min break between runs.

In the online experiment, each subject completed training and testing phases for the famous face and self-face spelling paradigms. In the training phase, there were four runs, and each run contained 20 sequences (whereby one character was revealed per sequence); that is, there were 20 characters in a run and a total of 80 characters in the training phase for each spelling paradigm, which were used to obtain the classifier. The test phase output a total of 30 characters by the trained classifier. In addition, trials were only repeated twice in each sequence for both the training and testing phases.

\section{Data Acquisition}

EEG signals were recorded with a NeuroScan amplifier (SynAmps 2, NeuroScan Inc., and Abbotsford, Australia). All signals were digitized at a rate of $250 \mathrm{~Hz}$, and band-pass filtered between 0.1 and $100 \mathrm{~Hz}$. Fourteen-channel (Fz, F3, F4, C3, Cz, C4, P7, P8, P3, P4, Pz, O1, Oz, and O2, Figure 3) EEG data were recorded with the $\mathrm{AFz}$ as the ground and the right mastoid as the reference electrode position. Horizontal eye movements were measured by deriving the electrooculogram (EOG) from a pair of horizontal EOG (HEOG) electrodes placed at the outer canthi of both the left and right eyes. Vertical eye movements and eye blinks were detected by deriving an EOG signal from a pair of vertical EOG (VEOG) electrodes placed $\sim 1 \mathrm{~cm}$ above and below the subject's left eye. The impedance was maintained below $5 \mathrm{~K} \Omega$.
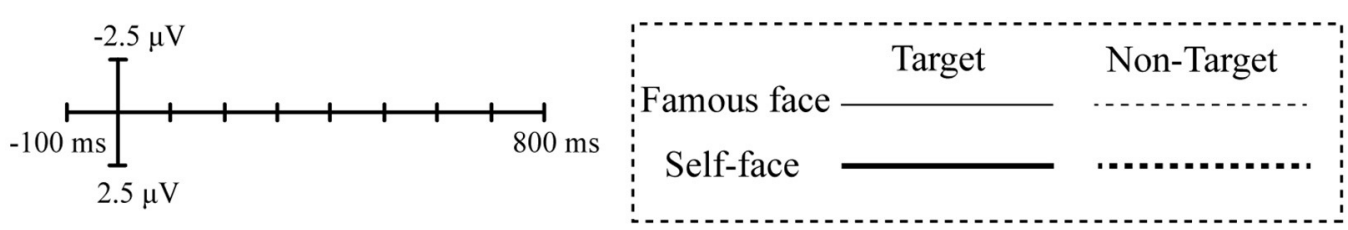

F3

$\mathrm{Fz}$

F4
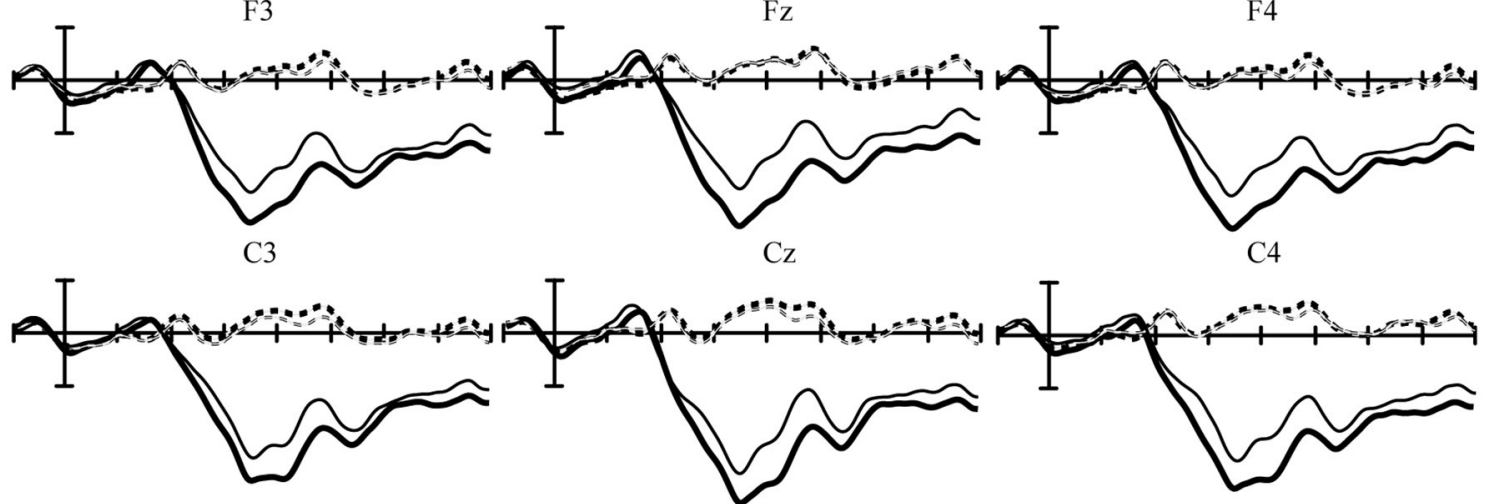

P3

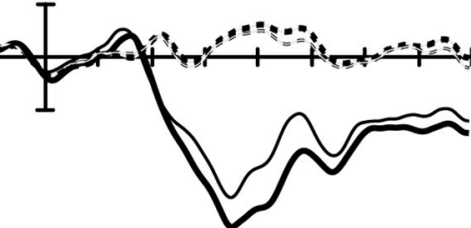

$\mathrm{Pz}$

$\mathrm{P} 4$
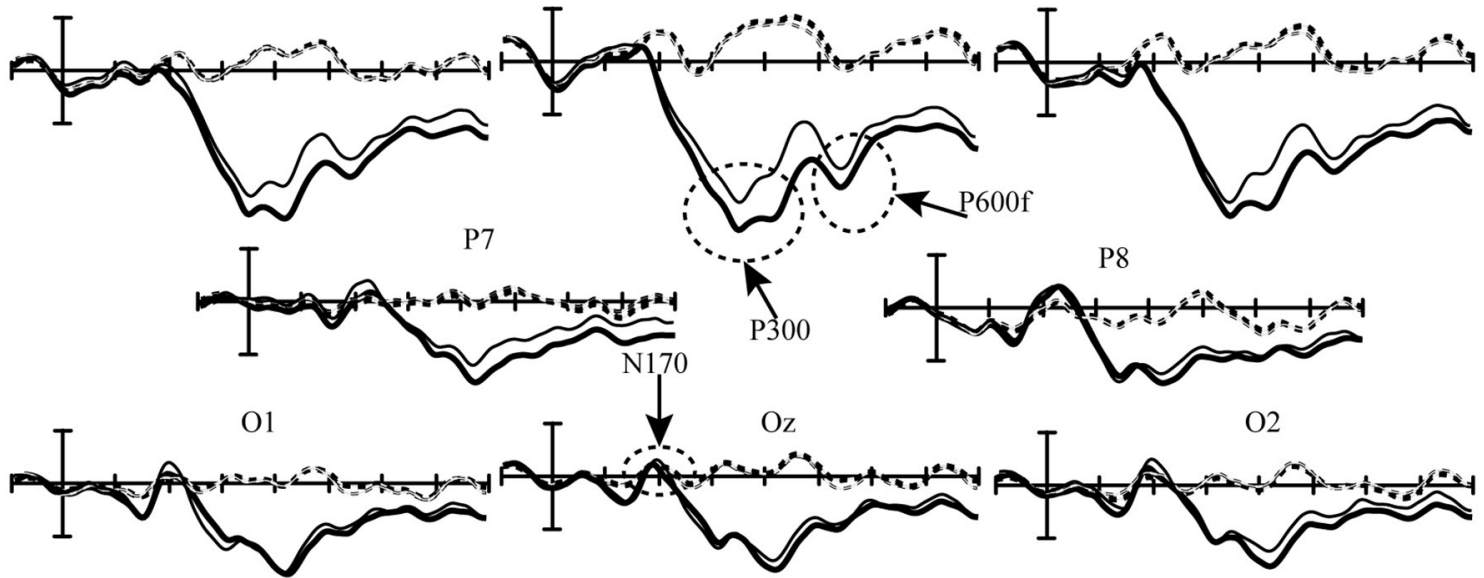

$\mathrm{O} 2$

FIGURE 4 | Superimposed grand-averaged event-related potentials elicited by the target and non-target stimuli over 14 electrodes in the self-face and famous face spelling paradigms. 

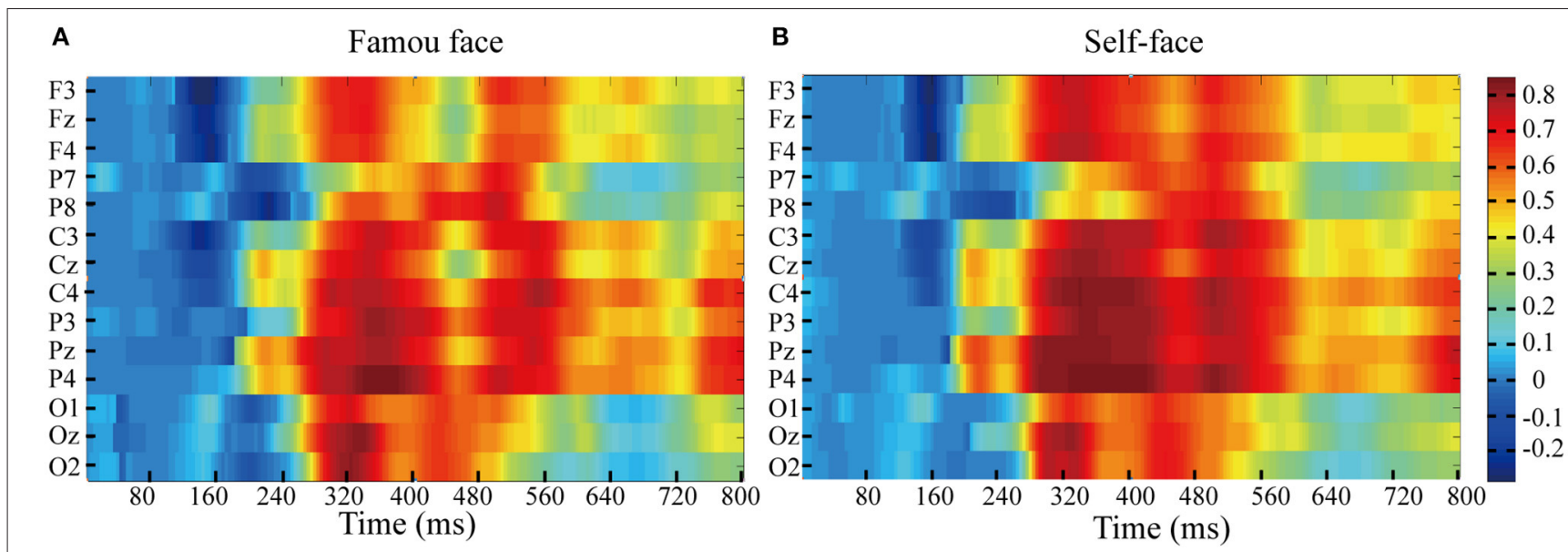

FIGURE $5 \mid R^{2}$ values of ERPs in response to the target and non-target stimuli between 0 and 800 ms from EEG data of all subjects in the famous face and self-face spelling paradigms. (A) $R^{2}$ values of ERPs for the famous face spelling paradigm. (B) $R^{2}$ values of ERPs for the self-face spelling paradigm.

\section{Feature Extraction Procedure}

For offline data, the classification performance of the speller depends not only on the amplitude of ERPs elicited by the target stimulus but also on the difference in ERP amplitudes elicited by the target and non-target stimuli. Thus, the analysis of $R^{2}$ values can provide the mathematic foundation for selecting channels and the features of each channel. The r-squared is calculated by formula (1)

$$
r^{2}=\left(\frac{\sqrt{N_{1} N_{2}}}{N_{1}+N_{2}} \times \frac{\operatorname{mean}\left(x_{1}\right)-\operatorname{mean}\left(x_{2}\right)}{\operatorname{std}\left(x_{1} \cup x_{2}\right)}\right)^{2}
$$

where $N_{1}$ and $N_{2}$ represent the sample size of the target and nontarget stimuli, respectively; $x_{1}$ and $x_{2}$ are features vector of the target and non-target stimuli, respectively.

According to the results of the r-squared values, ERP data of different time windows were down-sampled from 250 to $62.5 \mathrm{~Hz}$ by selecting every four samples, and the feature vector was $N_{p} \times N_{c}$, where $N_{p}$ represents the sample points within the selected time window, and $N_{c}$ represents the number of channels. For online data, the EEG data were first filtered between 0.1 and $30 \mathrm{~Hz}$ using a third-order Butterworth bandpass filter, then down-sampled from 250 to $50 \mathrm{~Hz}$. We extracted the EEG data from 200 to $800 \mathrm{~ms}$ after stimuli onset as the vector feature.

\section{Classification Scheme}

Bayesian linear discriminant analysis (BLDA) was used to classify the EEG data in the experiment. BLDA is an extension of Fisher's linear discriminant analysis that avoids over-fitting. The details of the algorithm have been described elsewhere (Hoffmann et al., 2008; Jin et al., 2014a). We used 4-fold cross-validation to calculate the individual accuracy in the offline experiment.

\section{Information Transfer Rate}

Information transfer rate (ITR) is generally used to evaluate the communication performance of a BCI system and is a standard measure that accounts for accuracy, the number of possible selections, and the time required to make each selection (Thompson et al., 2013). The ITR (bits $\mathrm{min}^{-1}$ ) can be calculated as follows:

$$
I T R=\frac{60\left(P \log _{2}(P)+(1-P) \log _{2} \frac{1-P}{N-1}+\log _{2} N\right)}{T}
$$

where $P$ denotes the probability of recognizing a character, $T$ is the time taken to recognize a character, and $N$ is the number of classes $(N=36)$.

\section{Data Analysis}

A one-way repeated measure ANOVA with the within-subjects two factors of spelling paradigm (self-face and famous face spelling paradigms) and electrodes (electrodes were based on the waveform of ERPs elicited by target stimuli) was used to compare the difference in ERP amplitudes between self-face and famous face spelling paradigms acquired by subtracting the waveforms elicited by non-target stimuli from that by target stimuli. The comparison of classification accuracy and ITR in offline and online experiments was conducted by a paired $T$-test. The statistical analyses were conducted using the SPSS version 19.0 software package (SPSS Inc., Chicago, IL, USA).

\section{RESULTS}

\section{ERP Results}

Figure 4 displays the superimposed grand-averaged waveform elicited by target and non-target stimuli in the self-face and famous face spelling paradigms. A clear negative peak was observed at $\mathrm{O} 1, \mathrm{Oz}$, and $\mathrm{Oz}$ between 150 and $200 \mathrm{~ms}$, which is indicative of the N170 potential. In addition, we observed a clear positive peak at all electrodes between 200 and $500 \mathrm{~ms}$, which is indicative of the $\mathrm{P} 300$ potential, and the other positive peak was observed between 500 and $600 \mathrm{~ms}$, at F3, Fz, F4, C3, Cz, C4, P3, $\mathrm{Pz}$, and $\mathrm{P} 4$, which is similar to the $\mathrm{P} 600 \mathrm{f}$ potential. 
A Fronto-central-parietal area

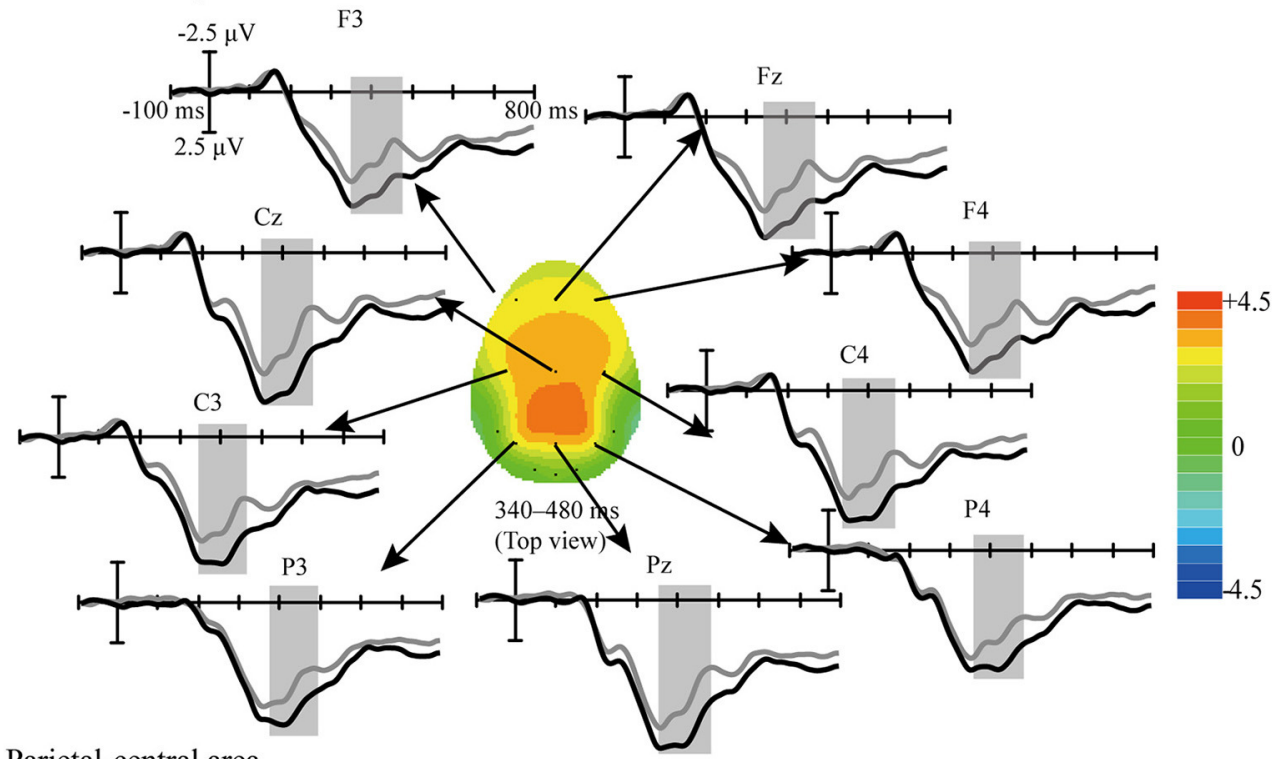

B Parietal-central area

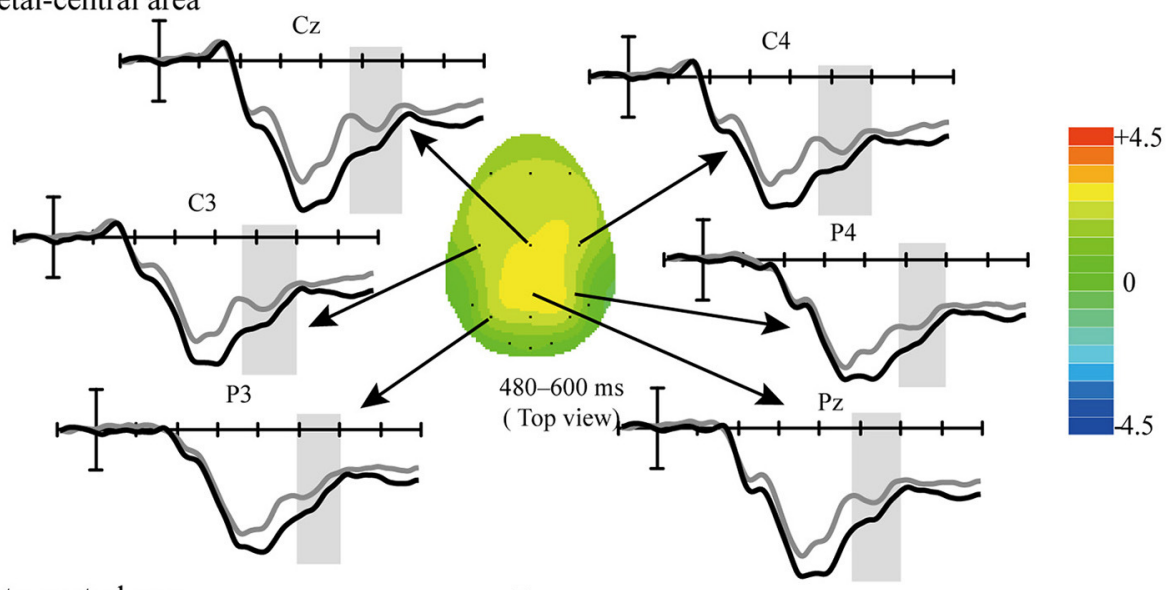

C Fronto-central area

$\mathrm{Fz}$

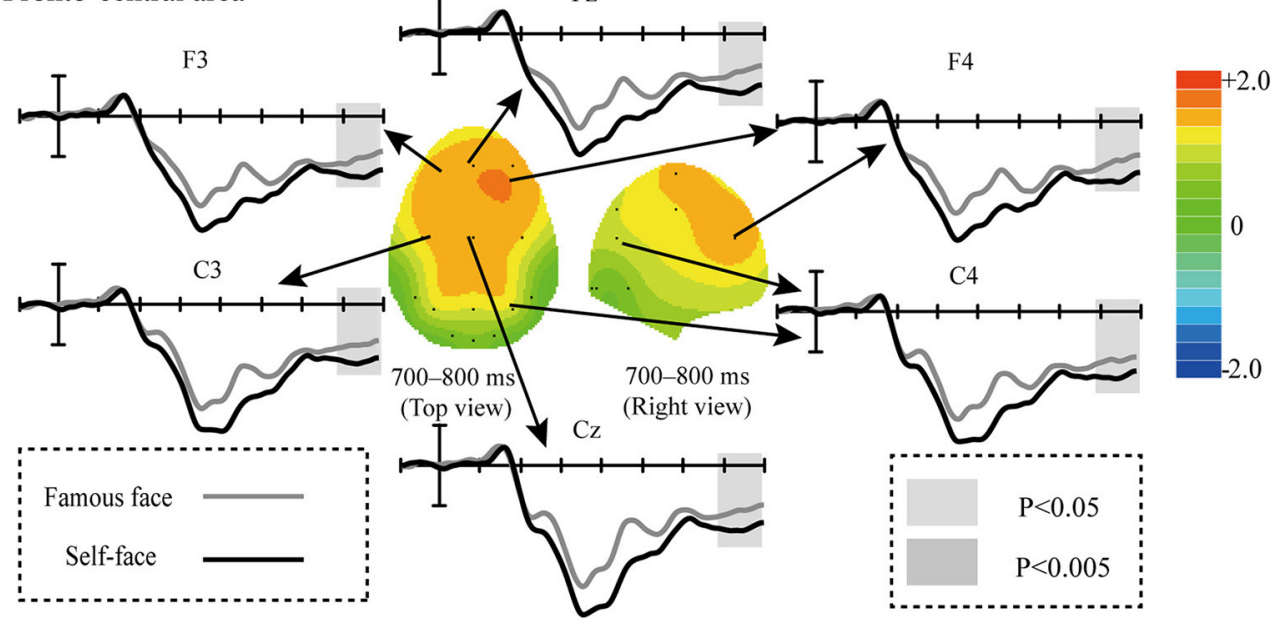

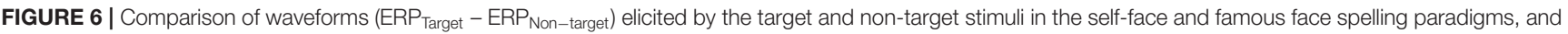
scalp topographies from difference waveforms. Difference waveforms were calculated by subtracting the ERPs of the famous face spelling paradigm from those of the self-face spelling paradigm. (A) The fronto-central-parietal area at $340-480 \mathrm{~ms}$. (B) The parietal-central area at 480-600 ms. (C) The fronto-central area at $700-800 \mathrm{~ms}$ 
Feature differences in the ERPs elicited by target and nontarget stimuli in the famous face and self-face spelling paradigms were indicated by the r-squared values (Figure 5). As seen in Figure 5, we observed that the feature differences in the ERPs elicited by target and no-target stimuli were mainly between 200 and $800 \mathrm{~ms}$ at all electrodes for both the famous face and selfface spelling paradigms. To represent the positive and negative deflections of ERP amplitude and to allow for richer visual information, we set the $R^{2}$ value corresponding to the negative ERP amplitude value as a negative value.

Figure 6 displays the scalp topographic regions that corresponded to significant differences between the waveforms elicited in the self-face and famous face spelling paradigms. Significant differences were observed in three regions corresponding to three time periods after stimulus presentation, as follows: the fronto-central-parietal area from 340 to $480 \mathrm{~ms}$ $\left[F_{(1,9)}=14.54, P<0.005\right.$; Figure 6A $]$; the parietal-central area from 480 to $600 \mathrm{~ms}\left[\mathrm{~F}_{(1,9)}=8.018, P<0.05\right.$; Figure $\left.6 \mathrm{~B}\right]$; and the fronto-central area from 700 to $800 \mathrm{~ms}\left[\mathrm{~F}_{(1,9)}=6.023\right.$, $P<0.05$; Figure 6C].

\section{Classification Results}

Based on the results of the r-squared values, we compared the classification accuracies based on two feature vectors, as follows: the feature vector A was $25 \times 12$ (time window of 200-700 ms, channels F3, Fz, F4, C3, Cz, C4, P3, Pz, P4, O1, Oz, and O4); the feature vector $\mathrm{B}$ was $45 \times 14$ (time window of $0-800 \mathrm{~ms}$, 14 channels). The results of classification accuracies based on feature A and feature B are shown in Figure 7, which shows the average accuracies across all subjects at each sequence in famous face and self-face spelling paradigms. There was no significant difference in accuracy between feature A and feature B in the two spelling paradigms.

Previous work has shown that the frequency band for the P300 is mainly between 1 and $10 \mathrm{~Hz}$ (Basar-Eroglu et al., 1992) and different band passes have been used to filter EEG data to acquire better classification accuracy, such as $1-4,1-12$, and $1-30 \mathrm{~Hz}$ (Jin et al., 2017). In this study, we compared the classification accuracies at the first three superpositions (superposition times represent the number of trials, that is, the repeating times of 6 rows/columns flashing) between $1-4,1-12$, and $1-30 \mathrm{~Hz}$ for the famous face and self-face spelling paradigms (Figure 8). We found that the average accuracy at $1-12 \mathrm{~Hz}$ was larger than that at $1-4 \mathrm{~Hz}$, and the average accuracy at $1-30 \mathrm{~Hz}$ was larger than that at $1-4 / 1-12 \mathrm{~Hz}$ for the first three superpositions in the two spelling paradigms except for the accuracies between 1-12 and 1$30 \mathrm{~Hz}$ at two superpositions in the famous face spelling paradigm. The paired $t$-test results revealed a significant difference for classification accuracy between $1-4$ and $1-12 \mathrm{~Hz} / 1-30 \mathrm{~Hz}$ in the famous face and self-face spelling paradigms.

Figure 9 shows the individual and average offline accuracies in the two face spelling paradigms based on the feature vector $B$ and a $1-30 \mathrm{~Hz}$ frequency band filter. The accuracies increased with the increase in the number of superpositions in both paradigms; the average spelling accuracy of the self-face spelling paradigm was greater than that in the famous face spelling paradigm at 115 superpositions. The average number of superpositions when

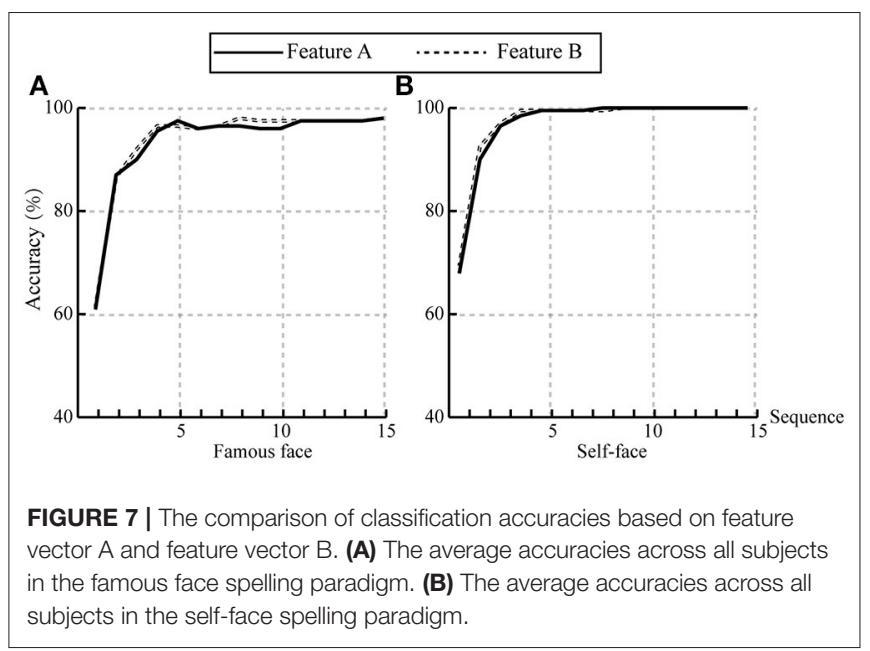

the accuracies reached $100 \%$ for all subjects was 2 in the self-face spelling paradigm; thus, we conducted a $t$-test on the accuracies only for the first two superpositions between the self-face and famous face paradigms. We found significant differences between the self-face and famous face spelling paradigms at both one superposition ( $t=-2.331, P<0.05$; Figure 10A) and two superpositions $(t=-2.25, P<0.05$; Figure 10B).

Table 1 shows the ITRs for each subject and the averages in the self-face and famous face spelling paradigms. The best ITR result, 31.4 bits $\min ^{-1}$ at one superposition, was found with the self-face spelling paradigm. The average ITR was greater at two superpositions than at one superposition. The paired $t$-tests showed that the ITR was significantly greater in the self-face paradigm than in the famous face paradigm at one superposition $(t=-2.414, P=0.039<0.05)$ and two superpositions $(t=$ $-2.345, P=0.044<0.05)$.

The online accuracies and ITRs of each subject for the famous face and self-face spelling paradigms are shown in Table 2. We found that the average accuracy and ITR in the selfface spelling paradigm were higher than those in the famous face spelling paradigm. Paired $t$-tests showed that there were significant differences in the accuracy and ITR between the two spelling paradigms (accuracy: $t=-2.643, P<0.05$; ITR: $t=$ $-3.140, P<0.05)$.

\section{DISCUSSION}

In the present study, we proposed a new P300-speller using selfface stimulus and assessed the grand-average ERP waveforms elicited by target stimuli in the new and control spelling paradigms, analyzed the different ERP waveforms and the scalp topographies corresponding to significantly different waveforms elicited by the target minus non-target stimuli, and compared the classification accuracy and ITR of offline and online experiments between the self-face and famous face spelling paradigms.

\section{ERPs}

Previous work has found that the performance of the P300-speller system could be improved by enhancing the 


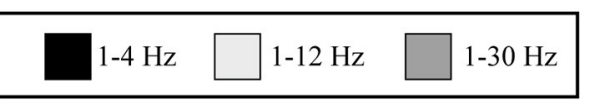

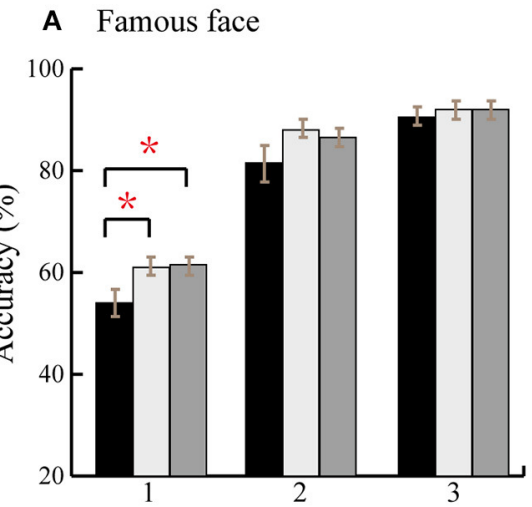

Supperposition time (s)
B Self-face

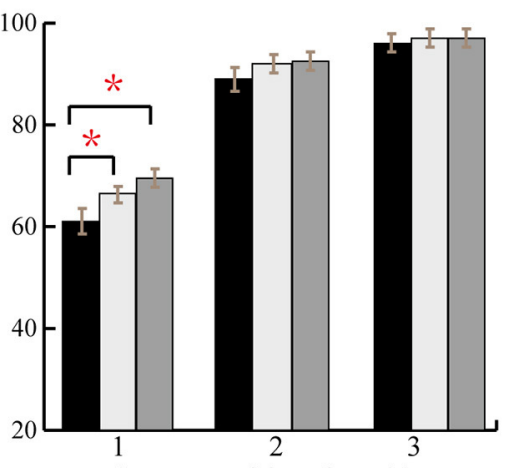

Supperposition time (s)

FIGURE 8 | Average offline classification accuracies across all subjects at the first three superpositions for 1-4, 1-12, and 1-30 Hz. (A) The comparison of accuracies between three frequency band filters in the famous face spelling paradigm. (B) The comparison of accuracies between three frequency band filters in the self-face spelling paradigm. *A significant difference in accuracy between two frequency bands.
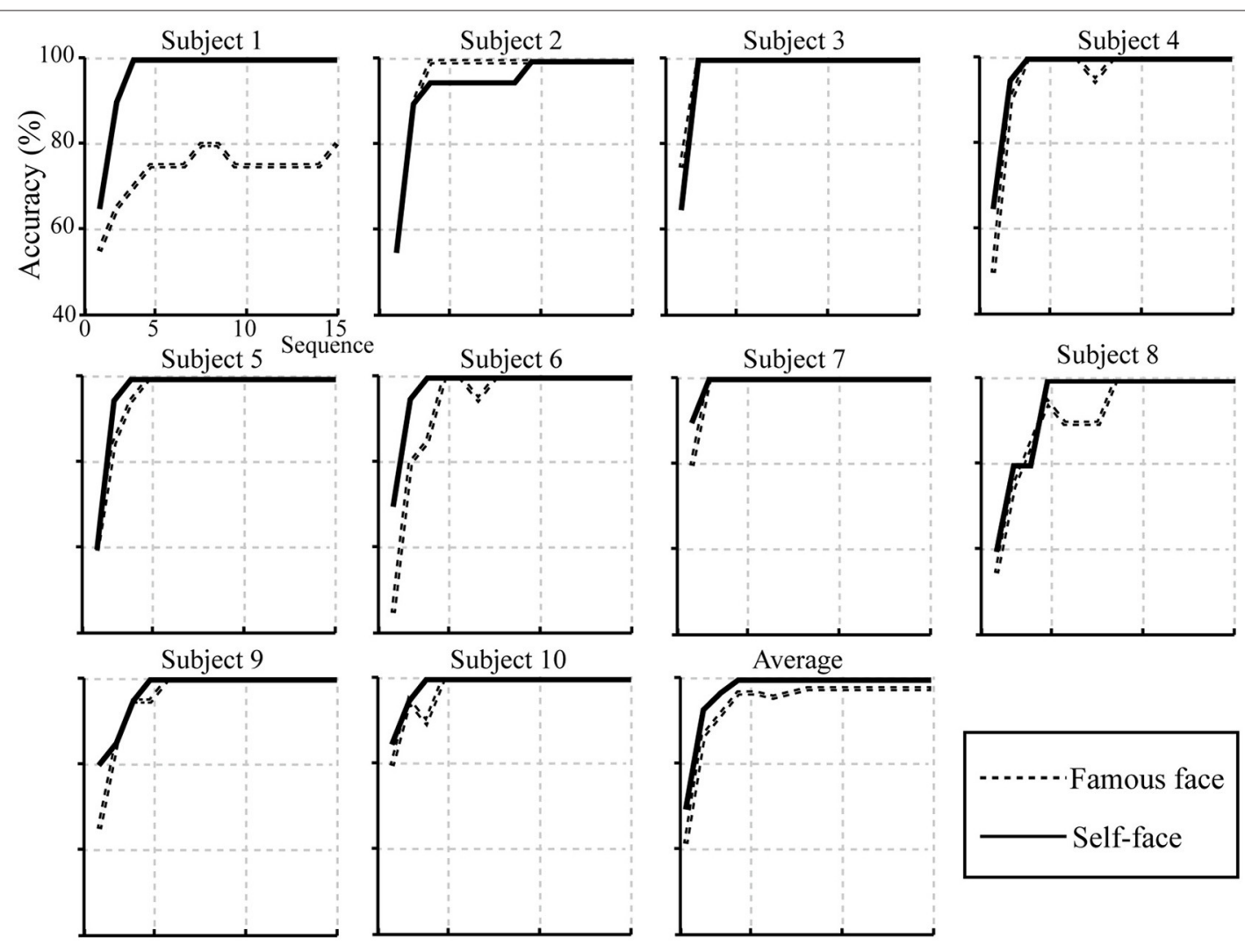

FIGURE 9 | Individual and average accuracies of the self-face and famous face spelling paradigms for 10 subjects.

difference between target trials and non-target trials (Jin et al., 2012). Therefore, we compared the waveforms $\left(\mathrm{ERP}_{\text {Target }}-\right.$ $\mathrm{ERP}_{\text {Non-target }}$ ) elicited during the two face paradigms and found a significant difference between the two. The first significantly different waveform was from 340 to $480 \mathrm{~ms}$ over the frontocentral-parietal area (Figure 6), i.e., the P300. The P300 is not only associated with attention and cognitive processing (Polich, 2007) but also reflects the involvement of higher-order cognitive 

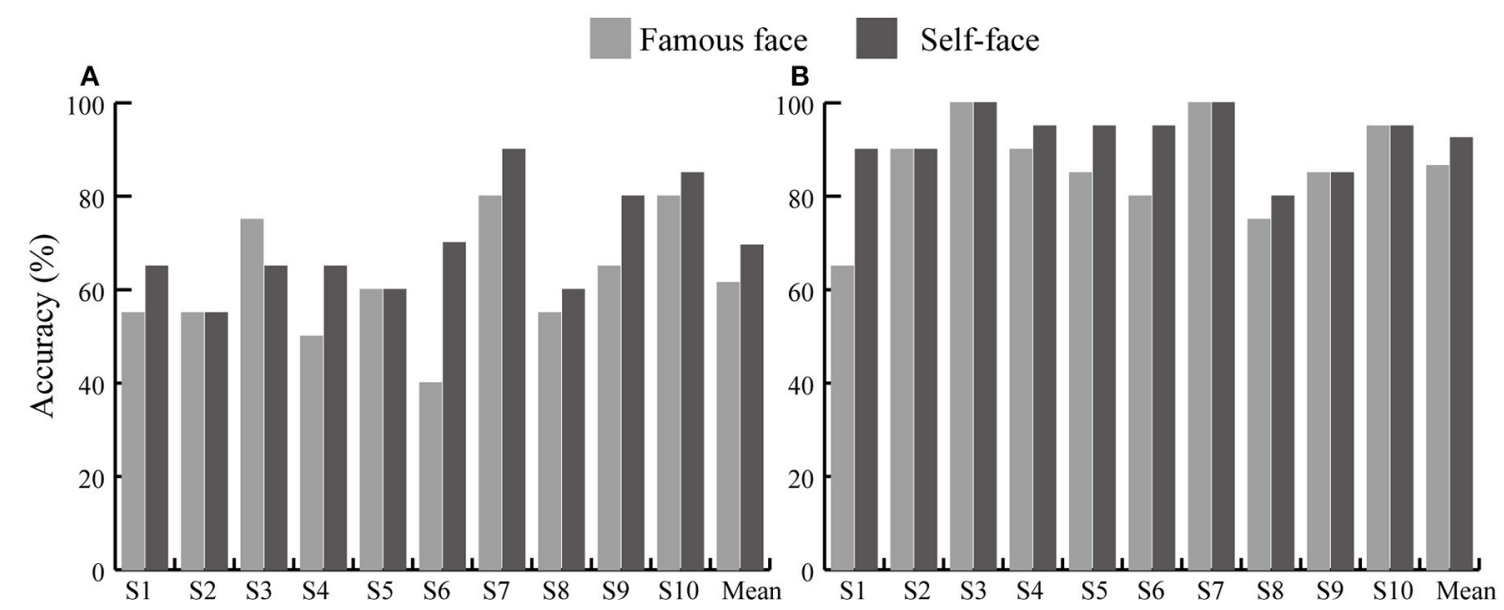

FIGURE 10 | Accuracies of each subject and mean accuracy of 10 subjects at one superposition and two superpositions for the famous face and self-face spelling paradigms. (A) Accuracies at one superposition. (B) Accuracies at two superpositions.

functions, including self-relevance (for one's own face, e.g., Ninomiya et al., 1998; Tanaka et al., 2006). Ninomiya et al. (1998) found that the P300 amplitude in response to one's own face was significantly larger than that in response to other stimuli. The authors, therefore, suggested that enhancement of the P300 in response to one's own face is not only due to an orienting response to a physically deviant stimulus but also due to the additional effect of relevance to the subject. Thus, the P300 can serve as an index of self-relevance, whereby higher selfrelevance corresponds to a larger P300 amplitude (Kok, 2001). In Miyakoshi et al.'s study, the P300 amplitude elicited by the self-face stimulus was greater than that elicited by a famous face, and the P300 could distinguish the self-face from a famous face, and the authors, therefore, suggested that the P300 amplitude was sensitive to self-relevance (Miyakoshi et al., 2008). Therefore, the larger amplitude P300 in the self-face spelling paradigm than in the famous face spelling paradigm may be due to the higher self-relevance of the self-face than of the famous face for subjects.

The second significant difference in positive waveform was observed from 480 to $600 \mathrm{~ms}$ at the parietal-central area (Figure 6); this was similar to the P600f, which is related to processes involved in the recollection of faces (Eimer, 2000; Curran and Hancock, 2007). Some studies have suggested that perception of an individual's face may induce spontaneous activation of the characteristic and information associated with the individual (Bargh et al., 1996; Todorov and Uleman, 2002). The ERPs between 500 and $700 \mathrm{~ms}$ with a larger amplitude in response to a familiar face as compared to an unfamiliar face may indicate that the perception of the familiar face automatically generated more of one's personal traits or other episodic information than the perception of an unfamiliar face (Sui et al., 2006). Curran and Hancock (2007) also reported that a familiar face elicited a larger positive waveform between 500 and $700 \mathrm{~ms}$ (P600f) than did a stranger's face. Thus, we speculate that the larger P600f amplitude observed in the selfface spelling paradigm than in the famous face spelling paradigm indicates that the self-face induced more recollection, including characteristic or episodic information about the self than did the famous face.

The third significant difference in positive waveforms was from 700 to $800 \mathrm{~ms}$ at the fronto-central area (Figure 6). In ERP studies of face recognition, attending to the self-face induced a larger amplitude waveform between 600 and $800 \mathrm{~ms}$ at the prefronto-central area than did attending to a familiar face; it was speculated that this component was affected by the allocation of attentional resources in face recognition (Sui et al., 2006). Miyakoshi et al. (2008) found that the self-face was more likely to attract the attention of participants than a familiar face. In our study, the increased amplitude between 700 and $800 \mathrm{~ms}$ for the self-face than for the famous face paradigm may indicate that subjects paid more attention to their own faces.

In addition, our results showed that there was no significant difference in the N170 amplitude between the two spelling paradigms. This may be due to differences in experimental design (Keyes et al., 2010; Alonso-Prieto et al., 2015). Alonso-Prieto et al. (2015) reported that the sensitivity of the N170 to faces with different levels of familiarity is affected by the experimental settings, such as faces with different facial angles or faces with emotional information. For example, there was a difference in the N170 between a famous face and the self-face in studies of the influence of facial angle (Miyakoshi et al., 2008) and of emotional expression (Caharel et al., 2005), while Tacikowski et al. (2011) found no difference in the N170 amplitude between the self-face and a famous face when using frontal and neutral face images. In our study, the famous face and self-face comprised frontal and neutral images; thus, our results are consistent with those of Tacikowski et al. In addition, the type of familiarity of the face has also been found to affect the sensitivity of the N170 (Alonso-Prieto et al., 2015). For example, Sui et al. (2006) found that the N170 did not differ between self-faces and familiar faces (classmates), while Keyes et al. (2010) showed an increased N170 amplitude to the self-face relative to familiar faces (good friends). 
TABLE 1 | The information transfer rate of each subject for the famous face and self-face spelling paradigms at one and two superpositions.

\begin{tabular}{llllll}
\hline & \multicolumn{2}{c}{ One superposition } & & \multicolumn{2}{c}{ Two superpositions } \\
\cline { 2 - 3 } \cline { 5 - 6 } Subject & Famous face & Self-face & & Famous face & Self-face \\
\hline Subject 1 & 14.0 & 18.3 & & 13.3 & 22.8 \\
Subject 2 & 14.0 & 14.0 & & 22.8 & 22.8 \\
Subject 3 & 23.1 & 18.3 & & 27.5 & 27.5 \\
Subject 4 & 12.0 & 18.3 & & 22.8 & 25.2 \\
Subject 5 & 16.1 & 16.1 & & 20.7 & 25.2 \\
Subject 6 & 8.4 & 20.6 & & 18.7 & 25.2 \\
Subject 7 & 25.7 & 31.4 & & 27.5 & 27.5 \\
Subject 8 & 14.0 & 16.1 & & 16.8 & 18.7 \\
Subject 9 & 18.3 & 25.7 & & 20.7 & 20.7 \\
Subject 10 & 25.7 & 28.4 & & 25.2 & 25.2 \\
Avg. \pm SD & $17.1 \pm 5.9$ & $20.7 \pm 5.8$ & $21.6 \pm 4.6$ & $24.1 \pm 2.8$ \\
P-value & $t=-2.414 ; p=0.039$ & & $t=-2.345 ; p=0.044$ \\
\hline
\end{tabular}

The unit of information transfer rate is bit/min.

TABLE 2 | The online accuracies and ITRs for all subjects in the famous face and self-face spelling paradigms.

\begin{tabular}{|c|c|c|c|c|}
\hline \multirow[b]{2}{*}{ Subject } & \multicolumn{2}{|c|}{ Accuracies (\%) } & \multicolumn{2}{|c|}{ ITRs (bit/min) } \\
\hline & Famous face & Self-face & Famous face & Self-face \\
\hline Subject 1 & 96.7 & 100.0 & 31.9 & 33.6 \\
\hline Subject 2 & 80.0 & 93.3 & 22.8 & 29.8 \\
\hline Subject 3 & 60.0 & 66.7 & 14.3 & 17.0 \\
\hline Subject 4 & 70.0 & 76.7 & 18.3 & 21.3 \\
\hline Subject 5 & 73.3 & 83.3 & 19.8 & 24.4 \\
\hline Subject 6 & 80.0 & 73.3 & 22.8 & 19.8 \\
\hline Subject 7 & 86.7 & 93.3 & 26.1 & 29.8 \\
\hline Subject 8 & 90.0 & 96.7 & 27.9 & 31.9 \\
\hline Subject 9 & 83.0 & 90.0 & 24.3 & 27.9 \\
\hline Subject 10 & 80.0 & 80.0 & 22.8 & 22.8 \\
\hline Avg. $\pm \mathrm{SD}$ & $80.0 \pm 10.4$ & $85.3 \pm 11.0$ & $23.1 \pm 5.0$ & $25.8 \pm 5.4$ \\
\hline$p$-value & \multicolumn{2}{|c|}{$t=-2.643, P<0.05$} & \multicolumn{2}{|c|}{$t=-3.140, P<0.05$} \\
\hline
\end{tabular}

In the present study, the reason we found no difference in the N170 between the two paradigms may be that the difference in familiarity level between the famous face (Ming Yao) and the selfface may not have been enough to induce a statistically significant difference in N170 amplitude.

\section{Classification Accuracies and ITR}

Offline classification results showed that the average accuracies of the self-face spelling paradigm were higher than those of the famous face spelling paradigm at all numbers of superpositions (Figure 9). A significant difference was found between the selfface and famous face spelling paradigm at one superposition $(P<0.05$; Figure 10A $)$ and at two superpositions $(P<$ 0.05; Figure 10B). The offline accuracies demonstrated that use of the self-face improved the performance of the facial spelling paradigm because the self-face stimulus induced larger ERP components than did the famous face. In addition, the improvement and stability of spelling accuracy required stimuli to be repeated several times because of the low signal-tonoise ratios; however, increasing the number of repetitions may reduce the spelling speed. Thus, the ITR depended on both classification accuracy and speed character output, which is an important statistical metric for the performance of the P300-speller system. Our results indicated that the ITR of the self-face spelling paradigm was significantly greater than that of the famous face spelling paradigm at the first two

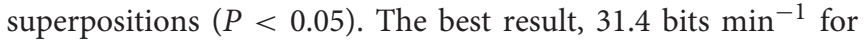
subject 7 , was obtained with the self-face spelling paradigm, in which subject 7 achieved $90 \%$ accuracy with one superposition only. Yet, the average ITR at two superpositions was larger than that at one superposition, and the standard deviation at one superposition was greater than that at two superpositions in both spelling paradigms (Table 1). This indicated that the spelling stability and performance is better at two superpositions. Therefore, in the online experiment, we set the trial to repeat only twice (that is, two superposition for 6 rows/columns) to acquire the accuracies and ITRs of character spelling. The online results showed that accuracy and ITR of the self-face spelling paradigm were significantly larger than those of the famous face spelling paradigm (Table 2 ). In summary, the proposed self-face spelling paradigm significantly improved the performance of the P300-speller system.

In addition, we compared the offline classification accuracies based on different feature vectors and frequency band passes. For feature vector A $(25 \times 12)$ and feature vector B $(45 \times 14)$, there was no significant difference at all superposition times, which indicates that the feature vector from amplitude difference between target and non-target stimuli can acquire classification results that are comparable to the feature vector in the $0-800 \mathrm{~ms}$ time window and at all channels (Figure 7). The classification results based on three frequency band passes showed that the best classification result was at $1-30 \mathrm{~Hz}$ at first three superpositions in both spelling paradigms (Figure 8), which indicated that a filter of $1-30 \mathrm{~Hz}$ could be a good choice for the classification accuracy of the P300-speller system.

\section{Future Work}

The analysis of ERPs, classification accuracies, and ITRs between the two spelling paradigms showed that the self-face stimulus elicited significantly increased ERP amplitudes compared to the famous face stimulus and improved the spelling accuracy and ITR of the P300-speller system. Moreover, the use of self-face also avoided the copyright issues caused by using a famous face. Thus, the proposed self-face paradigm promotes practical applications of BCIs system. Some recent studies have shown that the brain responded more positively to a happy face and which could elicit increased ERP amplitudes, compared to a neutral face stimulus (Denefrio et al., 2017; Lu et al., 2019). In future work, we intend to use the subject's own happy face to investigate whether the selfface with happy emotion can further improve the performance and practicability of the P300-speller system. 


\section{CONCLUSION}

This study investigated whether the use of the self-face could improve the performance of the P300-speller system as compared to the use of a famous face. We found a significant improvement in classification accuracy and ITR for the self-face spelling paradigm at the first two superpositions, as compared to the famous face spelling paradigm, which may have a significant impact on increasing the speed and accuracy of spelling. Moreover, this has significance in practical BCI applications because the use of a famous face may involve copyright infringement problems.

\section{DATA AVAILABILITY STATEMENT}

All datasets generated for this study are included in the article/supplementary material.

\section{ETHICS STATEMENT}

The studies involving human participants were reviewed and approved by the ethics committee of Changchun University of Science and Technology. The patients/participants provided their written informed consent to participate in this study. Written informed consent was obtained from the individual(s) for the

\section{REFERENCES}

Allison, B. Z., and Pineda, J. A. (2003). ERPs evoked by different matrix sizes: implications for a brain computer interface (BCI) system. IEEE Trans. Neural Syst. Rehabil. Eng. 11, 110-113. doi: 10.1109/TNSRE.2003.814448

Allison, B. Z., and Pineda, J. A. (2006). Effects of SOA and flash pattern manipulations on ERPs, performance, and preference: implications for a BCI system. Int. J. Psychophysiol. 59, 127-140. doi: 10.1016/j.ijpsycho.2005.02.007

Alonso-Prieto, E., Pancaroglu, R., Dalrymple, K. A., Handy, T., Barton, J. J., and Oruc, I. (2015). Temporal dynamics of the face familiarity effect: bootstrap analysis of single-subject event-related potential data. Cogn. Neuropsychol. 32, 266-282. doi: 10.1080/02643294.2015.1053852

Bargh, J. A., Chen, M., and Burrows, L. (1996). Automaticity of social behavior: direct effects of trait construct and stereotype-activation on action. J. Pers. Soc. Psychol. 71, 230-244. doi: 10.1037/0022-3514.71.2.230

Basar-Eroglu, C., Basar, E., Demiralp, T., and Schurmann, M. (1992). P300-response: possible psychophysiological correlates in delta and theta frequency channels. A review. Int. J. Psychophysiol. 13, 161-179. doi: 10.1016/0167-8760(92)90055-G

Bentin, S., and Deouell, L. Y. (2000). Structural encoding and identification in face processing: erp evidence for separate mechanisms. Cogn. Neuropsychol. 17, 35-55. doi: 10.1080/026432900380472

Bernat, E., Shevrin, H., and Snodgrass, M. (2001). Subliminal visual oddball stimuli evoke a P300 component. Clin. Neurophysiol. 112, 159-171. doi: 10.1016/S1388-2457(00)00445-4

Caharel, S., Courtay, N., Bernard, C., Lalonde, R., and Rebai, M. (2005). Familiarity and emotional expression influence an early stage of face processing: an electrophysiological study. Brain Cogn. 59, 96-100. doi: 10.1016/j.bandc.2005.05.005

Carbon, C. C., Schweinberger, S. R., Kaufmann, J. M., and Leder, H. (2005). The Thatcher illusion seen by the brain: an event-related brain potentials study. Brain Res. Cogn. Brain Res. 24, 544-555. doi: 10.1016/j.cogbrainres.2005.03.008

Carelli, L., Solca, F., Faini, A., Meriggi, P., Sangalli, D., Cipresso, P., et al. (2017). Brain-computer interface for clinical purposes: cognitive assessment and rehabilitation. Biomed. Res. Int. 2017:1695290. doi: 10.1155/2017/1695290 publication of any potentially identifiable images or data included in this article.

\section{AUTHOR CONTRIBUTIONS}

QL and ZL designed the experiment, wrote the manuscript, and revised the manuscript. ZL and NG implemented the experiment and accomplished the data processing. ZL and JY analyzed the experimental results and revised the manuscript. All authors read and approved the final manuscript.

\section{FUNDING}

This work was financially supported by the National Natural Science Foundation of China (grant numbers 61806025 and 61773076), Jilin Scientific and Technological Development Program (grant numbers 20190302072GX and 20180519012JH), and Scientific Research Project of Jilin Provincial Department of Education during the 13th Five-Year Plan Period (grant number JJKH20190597KJ).

\section{ACKNOWLEDGMENTS}

The authors further wish to thank all individuals who participated in our study.

Curran, T., and Hancock, J. (2007). The FN400 indexes familiarity-based recognition of faces. Neuroimage 36, 464-471. doi: 10.1016/j.neuroimage.2006.12.016

Denefrio, S., Simmons, A., Jha, A., and Dennis-Tiwary, T. A. (2017). Emotional cue validity effects: The role of neurocognitive responses to emotion. PLOS ONE 12:e0179714. doi: 10.1371/journal.pone.0179714

Eimer, M. (2000). Event-related brain potentials distinguish processing stages involved in face perception and recognition. Clin. Neurophysiol. 111, 694-705. doi: 10.1016/S1388-2457(99)00285-0

Farwell, L. A., and Donchin, E. (1988). Talking off the top of your head: toward a mental prosthesis utilizing event-related brain potentials. Electroencephalogr. Clin. Neurophysiol. 70, 510-523. doi: 10.1016/0013-4694(88)9 0149-6

Herzmann, G., Schweinberger, S. R., Sommer, W., and Jentzsch, I. (2004). What's special about personally familiar faces? A multimodal approach. Psychophysiology 41, 688-701. doi: 10.1111/j.1469-8986.2004.00196.x

Hoffmann, U., Vesin, J. M., Ebrahimi, T., and Diserens, K. (2008). An efficient P300-based brain-computer interface for disabled subjects. J. Neurosci. Methods 167, 115-125. doi: 10.1016/j.jneumeth.2007.03.005

Jeunet, C., Lotte, F., Batail, J. M., Philip, P., and Franchi, J. A. M. (2018). Using recent BCI literature to deepen our understanding of clinical neurofeedback: a short review. Neuroscience 378, 225-233. doi: 10.1016/j.neuroscience.2018.03.013

Jin, J., Allison, B. Z., Kaufmann, T., Kubler, A., Zhang, Y., Wang, X., et al. (2012). The changing face of P300 BCIs: a comparison of stimulus changes in a P300 BCI involving faces, emotion, and movement. PLoS ONE 7:e49688. doi: 10.1371/journal.pone.0049688

Jin, J., Allison, B. Z., Zhang, Y., Wang, X., and Cichocki, A. (2014a). An ERP-based BCI using an oddball paradigm with different faces and reduced errors in critical functions. Int. J. Neural Syst. 24:1450027. doi: 10.1142/S0129065714500270

Jin, J., Daly, I., Zhang, Y., Wang, X., and Cichocki, A. (2014b). An optimized ERP brain-computer interface based on facial expression changes. J. Neural. Eng. 11:036004. doi: 10.1088/1741-2560/11/3/0 36004 
Jin, J., Zhang, H., Daly, I., Wang, X., and Cichocki, A. (2017). An improved P300 pattern in BCI to catch user's attention. J. Neural Eng. 14:036001. doi: 10.1088/1741-2552/aa6213

Kaufmann, T., Schulz, S. M., Grunzinger, C., and Kubler, A. (2011). Flashing characters with famous faces improves ERP-based brain-computer interface performance. J. Neural Eng. 8:056016. doi: 10.1088/1741-2560/8/5/056016

Keyes, H., Brady, N., Reilly, R. B., and Foxe, J. J. (2010). My face or yours? Eventrelated potential correlates of self-face processing. Brain Cogn. 72, 244-254. doi: 10.1016/j.bandc.2009.09.006

Kircher, T. T., Senior, C., Phillips, M. L., Rabe-Hesketh, S., Benson, P. J., Bullmore, E. T., et al. (2001). Recognizing one's own face. Cognition 78, B1-B15. doi: $10.1016 / 50010-0277(00) 00104-9$

Kok, A. (2001). On the utility of $\mathrm{P} 3$ amplitude as a measure of processing capacity. Psychophysiology 38, 557-577. doi: 10.1017/S0048577201990559

Lazarou, I., Nikolopoulos, S., Petrantonakis, P. C., Kompatsiaris, I., and Tsolaki, M. (2018). EEG-based brain-computer interfaces for communication and rehabilitation of people with motor impairment: a novel approach of the 21 (st) century. Front. Hum. Neurosci. 12:14. doi: 10.3389/fnhum.2018. 00014

Li, Q., Liu, S., Li, J., and Bai, O. (2015). Use of a green familiar faces paradigm improves P300-speller brain-computer interface performance. PLoS ONE 10:e0130325. doi: 10.1371/journal.pone.0130325

Li, Q., Lu, Z., Gao, N., and Yang, J. (2019). Optimizing the performance of the visual P300-speller through active mental tasks based on color distinction and modulation of task difficulty. Front. Hum. Neurosci. 13:130. doi: 10.3389/fnhum.2019.00130

Lu, Z., Li, Q., Gao, N., Yang, J., and Bai, O. (2019). Happy emotion cognition of bimodal audiovisual stimuli optimizes the performance of the P300 speller. Brain Behav. 9:e01479. doi: 10.1002/brb3.1479

Miyakoshi, M., Kanayama, N., Nomura, M., Iidaka, T., and Ohira, H. (2008). ERP study of viewpoint-independence in familiar-face recognition. Int. J. Psychophysiol. 69, 119-126. doi: 10.1016/j.ijpsycho.2008.03.009

Ninomiya, H., Onitsuka, T., Chen, C. H., Sato, E., and Tashiro, N. (1998). P300 in response to the subject's own face. Psychiatry Clin. Neurosci. 52, 519-522. doi: 10.1046/j.1440-1819.1998.00445.x

Polich, J. (2007). Updating P300: an integrative theory of P3a and P3b. Clin. Neurophysiol. 118, 2128-2148. doi: 10.1016/j.clinph.2007.04.019

Rosenfeld, J. V., and Wong, Y. T. (2017). Neurobionics and the brain-computer interface: current applications and future horizons. Med. J. Aust. 206, 363-368. doi: $10.5694 / \mathrm{mja} 16.01011$

Salvaris, M., and Sepulveda, F. (2009). Visual modifications on the P300 speller BCI paradigm. J. Neural Eng. 6:046011. doi: 10.1088/1741-2560/6/4/046011

Schweinberger, S. R., Pickering, E. C., Jentzsch, I., Burton, A. M., and Kaufmann, J. M. (2002). Event-related brain potential evidence for a response of inferior temporal cortex to familiar face repetitions. Brain Res. Cogn. Brain Res. 14, 398-409. doi: 10.1016/S0926-6410(02)00142-8

Sellers, E. W., Krusienski, D. J., McFarland, D. J., Vaughan, T. M., and Wolpaw, J. R. (2006). A P300 event-related potential brain-computer interface (BCI): the effects of matrix size and inter stimulus interval on performance. Biol. Psychol. 73, 242-252. doi: 10.1016/j.biopsycho.2006.04.007

Speier, W., Deshpande, A., Cui, L., Chandravadia, N., Roberts, D., and Pouratian, N. (2017). A comparison of stimulus types in online classification of the P300 speller using language models. PLoS ONE 12:e0175382. doi: 10.1371 /journal.pone. 0175382
Sui, J., Zhu, Y., and Han, S. (2006). Self-face recognition in attended and unattended conditions: an event-related brain potential study. Neuroreport 17 , 423-427. doi: 10.1097/01.wnr.0000203357.65190.61

Tacikowski, P., Jednorog, K., Marchewka, A., and Nowicka, A. (2011). How multiple repetitions influence the processing of self-, famous and unknown names and faces: An ERP study. Int. J. Psychophysiol. 79, 219-230. doi: 10.1016/j.ijpsycho.2010.10.010

Takeuchi, N., Mori, T., Nishijima, K., Kondo, T., and Izumi, S. (2015). Inhibitory transcranial direct current stimulation enhances weak beta eventrelated synchronization after foot motor imagery in patients with lower limb amputation. J. Clin. Neurophysiol. 32, 44-50. doi: 10.1097/WNP.00000000000 00123

Tanaka, J. W., Curran, T., Porterfield, A. L., and Collins, D. (2006). Activation of preexisting and acquired face representations: the N250 event-related potential as an index of face familiarity. J. Cogn. Neurosci. 18, 1488-1497. doi: $10.1162 /$ jocn.2006.18.9.1488

Thompson, D. E., Blain-Moraes, S., and Huggins, J. E. (2013). Performance assessment in brain-computer interface-based augmentative and alternative communication. Biomed. Eng. Online 12:43. doi: 10.1186/1475-925X-12-43

Todorov, A., and Uleman, J. S. (2002). Spontaneous trait inferences are bound to actors' faces: evidence from a false recognition paradigm. J. Pers. Soc. Psychol. 83, 1051-1065. doi: 10.1037/0022-3514.83. 5.1051

Tong, F., and Nakayama, K. (1999). Robust representations for faces: evidence from visual search. J. Exp. Psychol. Hum. Percept. Perform. 25, 1016-1035. doi: 10.1037/0096-1523.25.4.1016

Townsend, G., LaPallo, B. K., Boulay, C. B., Krusienski, D. J., Frye, G. E., Hauser, C. K., et al. (2010). A novel P300-based brain-computer interface stimulus presentation paradigm: moving beyond rows and columns. Clin. Neurophysiol. 121, 1109-1120. doi: 10.1016/j.clinph.2010. 01.030

Waldert, S. (2016). Invasive vs. non-invasive neuronal signals for brain-machine interfaces: will one prevail? Front. Neurosci. 10:295. doi: 10.3389/fnins.2016.00295

Wang, F., He, Y., Qu, J., Cao, Y., Liu, Y., Li, F., et al. (2019). A brain-computer interface based on three-dimensional stereo stimuli for assisting clinical object recognition assessment in patients with disorders of consciousness. IEEE Trans. Neural. Syst. Rehabil. Eng. 27, 507-513. doi: 10.1109/TNSRE.2019.28 96092

Wang, F., He, Y., Qu, J., Xie, Q. Y., Lin, Q., Ni, X. X., et al. (2017). Enhancing clinical communication assessments using an audiovisual BCI for patients with disorders of consciousness. J. Neural Eng. 14:046024. doi: 10.1088/1741-2552/aa6c31

Conflict of Interest: The authors declare that the research was conducted in the absence of any commercial or financial relationships that could be construed as a potential conflict of interest.

Copyright () $2020 \mathrm{Lu}, \mathrm{Li}, \mathrm{Gao}$ and Yang. This is an open-access article distributed under the terms of the Creative Commons Attribution License (CC BY). The use, distribution or reproduction in other forums is permitted, provided the original author(s) and the copyright owner(s) are credited and that the original publication in this journal is cited, in accordance with accepted academic practice. No use, distribution or reproduction is permitted which does not comply with these terms. 